\title{
Shear viscosity and imperfect fluidity in bosonic and fermionic superfluids
}

\author{
Rufus Boyack ${ }^{1}$, Hao Guo ${ }^{2}$ and K. Levin ${ }^{1}$ \\ ${ }^{1}$ James Franck Institute, University of Chicago, Chicago, Illinois 6063\%, USA and \\ ${ }^{2}$ Department of Physics, Southeast University, Nanjing 211189, China
}

\begin{abstract}
In this paper we address the ratio of the shear viscosity to entropy density $\eta / s$ in bosonic and fermionic superfluids. A small $\eta / s$ is associated with nearly perfect fluidity, and more general measures of the fluidity perfection/imperfection are of wide interest to a number of communities. We use a Kubo approach to concretely address this ratio via low temperature transport associated with the quasi-particles. Our analysis for bosonic superfluids utilizes the framework of the oneloop Bogoliubov approximation, whereas for fermionic superfluids we apply BCS theory and its BCS-BEC extension. Interestingly, we find that the transport properties of strict BCS and Bogoliubov superfluids have very similar structures, albeit with different quasi-particle dispersion relations. While there is a dramatic contrast between the power law and exponential temperature dependence for $\eta$ alone, the ratio $\eta / s$ for both systems is more similar. Specifically we find the same linear dependence (on the ratio of temperature $T$ to inverse lifetime $\gamma(T)$ ) with $\eta / s \propto T / \gamma(T)$, corresponding to imperfect fluidity. By contrast, near the unitary limit of BCS-BEC superfluids a very different behavior results, which is more consistent with near-perfect fluidity.
\end{abstract}

PACS numbers: $72.10 . \mathrm{Bg}, 74.25 . \mathrm{fc}$

\section{INTRODUCTION}

There is a growing interest among particle, condensed matter and atomic physicists on the behavior of the shear viscosity $\eta$ and the degree to which the ratio $\eta / s$, where $s$ is the entropy density, is close to the lower bound $\eta / s \geq \hbar /\left(4 \pi k_{B}\right)$ conjectured by Kovtun, Son, and Starinets (KSS) [1]. The KSS conjecture has led to renewed interest as to which fluids in nature are "perfect" fluids, i.e., those that come as close as possible to minimizing the conjectured bound.

For atomic Fermi gases there have been experimental $[2,3]$ and theoretical [4] studies of this ratio which suggest a close approach to the KSS bound. These have been in the specific unitary regime; no such studies are yet available for the bosonic superfluids. Theories of $\eta / s$ in graphene [5], along with high $T_{C}$ superconductor experiments [6], have also made claims that these exhibit an $\eta / s$ near the KSS bound.

It is of interest then to perform concrete calculations of this ratio, particularly in the presence of the many body physics which gives rise to superfluidity. Thus, in this paper we address the quasi-particle contribution to $\eta / s$ for bosonic and fermionic superfluids. We arrive at a fairly generic behavior for this ratio of the form $\eta / s \propto T / \gamma(T)$, where $\gamma(T)$ is the temperature dependent inverse quasi-particle lifetime. While classical arguments have led to a prediction of this form, we show that it holds as well for the extreme non-classical regimes in systems with widely different quasi-particle dispersion relations. A scaling of $\eta / s$ of this form has also been argued to apply near quantum critical points, or for nodal $d$-wave superfluids, and in these contexts, it has been argued that the behavior is suggestive of near perfect fluidity [6].

More generally, here we present a comparison of the dissipative transport properties of bosonic and fermionic superfluids mostly at low temperatures, where bosonic superfluid theories exist and are controlled. For the fermionic superfluids there is no restriction on temperature, and one can, furthermore, probe the behavior of an interesting normal phase (with a pseudogap) in the regime of BCS-BEC. In addition to the shear viscosity $\eta$, we focus on the quasi-particle contributions to transport (which are the exclusive contributions) in the thermal conductivity $\kappa$, the $\omega \neq$ 0 mass conductivity $\sigma$, and the off-diagonal thermoelectric coefficients. These are to be distinguished from condensate contributions, which dominate the $\omega \equiv 0$ mass conductivity. For the latter, a proper theory of transport has to deal with a number of subtle features involving gauge invariance and the important constraint in bosonic transport in which the (two particle) density excitation spectrum or sound modes are intimately coupled to the single particle excitations. Our starting point for bosonic superfluids is due to Wong and Gould [7], and Talbot and Griffin [8], and known as the "oneloop approximation".

We note that prior to the recent focus on trapped atomic gas superfluids there were puzzles concerning the behavior of the shear viscosity alone, which originated in contrasting observations of the fermionic and bosonic counterparts of liquid helium. For example, ${ }^{3} \mathrm{He}$ and ${ }^{4} \mathrm{He}$ exhibit a remarkable difference in their shear viscosity for low temperatures. At temperatures below the critical temperature the shear viscosity of fermionic ${ }^{3} \mathrm{He}$ has been measured [9] to be a decreasing function of decreasing temperature, whereas the shear viscosity of bosonic ${ }^{4} \mathrm{He}$ 
[10] is an increasing function of decreasing temperature. In this paper we suggest that these differences are understood as reflecting the different dispersions of the quasiparticles. Indeed, a central theme of this work is that the shear viscosity itself provides a sensitive measure of the nature of the quasi-particle excitations.

Our calculations show that the one-loop Bogoliubov theory for bosons and the BCS theory for fermions are formally strikingly similar. Nevertheless, primarily as a result of differences in the quasi-particle excitation spectrum, as well as the statistics, there are important differences in superfluid transport. Quite generally, in bosonic systems, because the dispersion relation is gapless, the transport coefficients increase more rapidly as a function of temperature when compared to the gapped fermionic systems. This results in low temperature transport in the bosonic case being more accessible experimentally.

\section{THEORY}

Previous studies of superfluid transport have relied heavily on kinetic theory and a Boltzmann equation ansatz $[11,12]$. A less widely applied approach has been the use of linear response theory and Kubo formulae, which we will use here. The advantage of the Kubo formulae approach is that by relating directly to Green's function diagrammatics one has better control over the processes included in transport and the appropriate constraints. This enables a more systematic imposition of perturbation expansions, which is especially crucial when considering bosonic superfluids. Also important are the constraints which must be imposed on separating the contributions associated with longitudinal and transverse correlations, since it is only in the former that the condensate will directly enter. Finally, a subtle but important issue here arises in the shear viscosity, for example, where the Kubo formula shows that there are multiple response functions which enter in addition to the simplest stress tensor-stress tensor correlator [13]. It is not as apparent how to include these in a Boltzmann based approach.

Since it is likely that dissipation in the ultracold gases is linked to the details of the experimental set up, we will introduce dissipation via a phenomenological parametrization within the Kubo approach. The philosophy behind our phenomenological approach to dissipation is similar to that articulated by Kadanoff and Martin, who emphasized the importance of the Kubo based correlation functions and their symmetries [14]. In related work on superconductors [15], they argued for the suitability of introducing a parametrization of the lifetimes associated with transport. In building any phenomenology it is important to emphasize that interparticle collisions can not be the sole source of dissipation in mass transport, as in the particle conductivity. This particular transport coefficient reflects the fact that the total momentum would be conserved (in the presence of Galilean invariance) without other sources of momentum relaxation.

By contrast, the strength of Boltzmann theory is that if all the details of the processes giving rise to dissipation are well established, one can incorporate dissipation via specific collision integrals. Within a Boltzmann based theory of bosonic superfluid transport, there is a fairly extensive review by Griffin [12] in which the shear viscosity and the thermal conductivity are addressed. At a qualitative level our Kubo calculations are consistent with this earlier work, but we also include additional transport coefficients. A Kubo formulation of the shear viscosity of a trapped Bose condensed gas was studied in [16] within the second order Beliaev approximation, but this analysis did not incorporate the contribution from anomalous Green's functions. This paper revisits this earlier work (albeit without a trap) and with the important inclusion of the anomalous Green's functions, which are a crucial component for a consistent treatment of superfluid transport.

\section{Physical Analysis of the Quasi-particle Regime}

In order to further understand the role of the phenomenological inverse lifetime, we address $\eta / s$ using a simple classical argument [17]. The entropy density $s$ of a weakly interacting system is proportional to the quasi-particle number density $n$ :

$$
s \sim k_{B} n .
$$

The shear viscosity is proportional to the product of the average energy per particle $\epsilon$, and the mean free time between collisions $\tau \equiv 1 / \gamma$ :

$$
\eta \sim n \epsilon / \gamma .
$$

Then, assuming $\epsilon \sim k_{B} T$, the ratio $\eta / s$ is

$$
\eta / s \sim T / \gamma .
$$

In order for the quasi-particle picture to be valid, the particles must be long lived: $\hbar \gamma<<k_{B} T$ so that the ratio $\eta / s$ is far above the KSS bound:

$$
\eta / s>>\hbar / 4 \pi k_{B}
$$

Importantly, we will show in this paper that, even in the non-classical regime, for both bosonic and fermionic superfluids, an equation of the form given in Eq. (1) results. In this way the quasi-particle regime should be understood as a regime where the system is far from being a perfect fluid. 


\section{Overview of Our Transport Results}

We begin by summarizing our results, which serve to emphasize the formal similarity of the bosonic oneloop transport theory with the fermionic BCS transport theory. We define the general transport coefficients $\left(L_{i j}\right)$ via particle $\left(\mathbf{J}_{p}\right)$ and heat $\left(\mathbf{J}_{Q}\right)$ current densities as follows

$$
\begin{aligned}
\mathbf{J}_{p} & =-L_{11} \nabla \mu-L_{12} \nabla T, \\
\mathbf{J}_{Q} & =-L_{21} \nabla \mu-L_{22} \nabla T,
\end{aligned}
$$

where $\nabla \mu$ and $\nabla T$ represent imposed gradients of the chemical potential (analogous to the electric field for a charged system) and the temperature. (We work in units where $\hbar=k_{B}=e=1$.) Here the particle or mass conductivity $\sigma \equiv L_{11}$ and the thermal conductivity $\kappa \equiv$ $L_{22}$. The off-diagonal transport coefficients appear, for example, in the quasi-particle thermopower.

For a superfluid it should be stressed that the various correlation functions that enter into the $L_{i j}$ may be distinct for longitudinal and transverse properties. This distinction is most important for the mass conductivity, as the longitudinal contribution reflects the condensate (and diverges at zero momentum and frequency) while the transverse contribution reflects the quasi-particles. The shear viscosity is also represented in terms of this transverse response.

Following the approach of Kadanoff and Martin [15], lifetime effects are phenomenologically incorporated by introducing the parameter $\gamma(T)$. In this context $\gamma^{-1}$ was introduced as a lifetime required to restore local equilibrium to a system perturbed from the equilibrium state. It may therefore be regarded as an additional experimental parameter for the particular system of interest. In the context of superfluids $\gamma^{-1}$ can be associated with quasi-particle lifetime processes, which in certain cases are known [11].

Using the correlation functions which will appear in Eqs. (16-19) below, we find that, for bosons, the transport coefficients (in $3 \mathrm{~d}$ ) are

$$
\begin{aligned}
\eta^{B} & =\int_{0}^{\infty} d k \frac{k^{6}}{30 \pi^{2} m^{2}}\left(\frac{\xi_{\mathbf{k}}}{E_{\mathbf{k}}}\right)^{2}\left(-\frac{\partial n\left(E_{\mathbf{k}}\right)}{\partial E_{\mathbf{k}}}\right) \frac{1}{\gamma}, \\
\operatorname{Re} L_{i j}^{B} & =T^{1-j} \int_{0}^{\infty} d k \frac{k^{4}}{6 \pi^{2} m^{2}} \xi_{\mathbf{k}}^{i+j-2}\left(-\frac{\partial n\left(E_{\mathbf{k}}\right)}{\partial E_{\mathbf{k}}}\right) \frac{1}{\gamma} .
\end{aligned}
$$

Note we have evaluated $\eta^{B}, \operatorname{Re} L_{i j}^{B}$ in the limit $\omega \rightarrow 0$.

We introduce $n_{0}$ as the condensate density and $g$ as the interaction strength. The Hugenholtz-Pines theorem determines the chemical potential, in the Bogoliubov approximation, as $\mu^{B}=n_{0} g$. The free particle dispersion relation is $\epsilon_{\mathbf{k}}=\frac{k^{2}}{2 m}$ and we define $\xi_{\mathbf{k}}=\epsilon_{\mathbf{k}}+\mu^{B}$. The Bogoliubov quasi-particle dispersion relation is then
$E_{\mathbf{k}}^{2}=\xi_{\mathbf{k}}^{2}-\left(\mu^{B}\right)^{2}$. We define $n(x)=\left[e^{x / T}-1\right]^{-1}$ as the Bose-Einstein distribution function.

The same calculations performed above for bosons can be performed for strict BCS fermions. The only differences that arise are a sign factor due to the different statistics, a degeneracy factor of two due to spin and a redefinition of the dispersion relation. For fermions the transport coefficients are

$$
\begin{aligned}
\eta^{F} & =\int_{0}^{\infty} d k \frac{k^{6}}{15 \pi^{2} m^{2}}\left(\frac{\xi_{\mathbf{k}}}{E_{\mathbf{k}}}\right)^{2}\left(-\frac{\partial f\left(E_{\mathbf{k}}\right)}{\partial E_{\mathbf{k}}}\right) \frac{1}{\gamma}, \\
\operatorname{Re} L_{i j}^{F} & =T^{1-j} \int_{0}^{\infty} d k \frac{k^{4}}{3 \pi^{2} m^{2}} \xi_{\mathbf{k}}^{i+j-2}\left(-\frac{\partial f\left(E_{\mathbf{k}}\right)}{\partial E_{\mathbf{k}}}\right) \frac{1}{\gamma} .
\end{aligned}
$$

where $\xi_{\mathbf{k}}=\epsilon_{\mathbf{k}}-\mu^{F}, E_{\mathbf{k}}^{2}=\xi_{\mathbf{k}}^{2}+\Delta^{2}$, and $f(x)=$ $\left[e^{x / T}+1\right]^{-1}$. (We have again evaluated $\eta^{F}, \operatorname{Re} L_{i j}^{F}$ in the limit $\omega \rightarrow 0$.) This expression for the shear viscosity has been obtained previously in [4]. Similarly the mass and thermal conductivities $L_{11}, L_{22}$ are consistent with results obtained from BCS theory [15]. Our emphasis here is that a comparison between Eqs. (4-5) and Eqs. (6-7) shows the striking similarities between the transport coefficients in bosonic and fermionic superfluids. A key difference between the transport coefficients arises from the soft quasi-particle excitations for bosons as opposed to the gapped excitations for fermions.

\section{Details of the Derivation}

We proceed now to derive Eqs. (4-7). In linear response theory the response of a system perturbed slightly from thermal equilibrium is expressed in terms of correlation functions of the unperturbed system [14]. Equations (2-3) lead to four possible correlation functions involving combinations of particle and heat or energy currents. These four correlation functions are

$$
\overleftrightarrow{\chi}_{i j}\left(x_{1}-x_{2}, \tau_{1}-\tau_{2}\right)=-\left\langle T_{\tau} j_{i}\left(x_{1}, \tau_{1}\right) j_{j}\left(x_{2}, \tau_{2}\right)\right\rangle,
$$

where $i, j \in\{1,2\}$. The particle and heat currents which appear above are defined as [15]

$$
\begin{aligned}
& j_{1}=-\left.\frac{i}{2 m}\left(\nabla_{1}-\nabla_{1}^{\prime}\right) \psi^{+}\left(1^{\prime}\right) \psi(1)\right|_{1^{\prime}=1^{+}}, \\
& j_{2}=-\left.\frac{i}{2 m}\left(\partial_{t_{2}} \nabla_{2}^{\prime}+\partial_{t_{2}}^{\prime} \nabla_{2}\right) \psi^{+}\left(2^{\prime}\right) \psi(2)\right|_{2^{\prime}=2^{+}}
\end{aligned}
$$

For a generic superfluid correlation function $\overleftrightarrow{\chi}_{i j}$, it is convenient to decompose into longitudinal and transverse components which are given by $\chi_{i j}^{L}=\frac{\mathbf{q} \cdot \overleftrightarrow{\chi}_{i j} \cdot \mathbf{q}}{q^{2}}, \chi_{i j}^{T}=$ $\frac{1}{2}\left(\sum_{\alpha} \chi_{i j}^{\alpha \alpha}-\chi_{i j}^{L}\right)$.

We define the Fourier transform by $\overleftrightarrow{\chi}_{i j}\left(x_{1}-x_{2}, \tau_{1}-\right.$ $\left.\tau_{2}\right)=\frac{1}{\beta} \sum_{i \omega_{m}} \int \frac{d^{3} q}{(2 \pi)^{3}} \overleftrightarrow{\chi}_{i j}\left(\mathbf{q}, i \omega_{m}\right) e^{i \mathbf{q} \cdot\left(\mathbf{x}_{1}-\mathbf{x}_{2}\right)} e^{-i \omega_{m}\left(\tau_{1}-\tau_{2}\right)}$. 
Then the Kubo formulas for the transport coefficients, except those associated with $\chi_{11}$, are

$$
\operatorname{Re} L_{i j}=-T^{1-j} \lim _{\mathbf{q} \rightarrow 0} \frac{\operatorname{Im} \chi_{i j}^{L}(\mathbf{q}, \omega)}{\omega}, \quad i, j \neq 1 .
$$

Using this definition, one can compute transport coefficients $\operatorname{Re} L_{i j}^{B}, i, j \neq 1$ for the bosonic case and $\operatorname{Re} L_{i j}^{F}, i, j \neq 1$ for the fermionic case.

The quasi-particle contribution to the mass conductivity and the shear viscosity (for which there is no condensate component) depend only on the transverse component of $\overleftrightarrow{\chi}_{11}$ and are given by [14]

$$
\begin{aligned}
\operatorname{Re} \sigma(\omega \neq 0) & =-\lim _{\mathbf{q} \rightarrow 0} \frac{\operatorname{Im} \chi_{11}^{T}(\mathbf{q}, \omega)}{\omega}, \\
\eta & =-m^{2} \lim _{\omega \rightarrow 0} \lim _{\mathbf{q} \rightarrow 0} \frac{\omega}{q^{2}} \operatorname{Im} \chi_{11}^{T}(\mathbf{q}, \omega) .
\end{aligned}
$$

By limiting consideration in $\sigma$ to $\omega \neq 0$, we focus on the quasi-particle transport. The total mass conductivity (which includes the condensate) is $\operatorname{Re} \sigma(\omega)=\operatorname{Re} \sigma(\omega \neq$ $0)+\frac{\pi n_{s}}{m} \delta(\omega)$, where $\frac{n_{s}}{m}$ is the superfluid density. The mass conductivity of the condensate is infinite but all condensate thermoelectric coefficients vanish. More specifically the condensate enters directly into only $L_{11}$. Finally, we note that the Onsager relation between the associated transport coefficients is $L_{12}=L_{21} / T$.

\section{Bosonic One Loop Approximation and Correlation Functions}

In order to evaluate the various $\overleftrightarrow{\chi}_{i j}$ we introduce the appropriate Green's functions. These functions are well established for the case of fermionic BCS superfluids. For the bosonic case, the one-loop approximation is based on the Bogoliubov Green's functions and thus involves the Bogoliubov quasi-particle dispersion relation. The Green's functions in the Bogoliubov approximation are

$$
\begin{aligned}
& G(K)=\frac{u_{\mathbf{k}}^{2}}{i \omega_{n}-E_{\mathbf{k}}}-\frac{v_{\mathbf{k}}^{2}}{i \omega_{n}+E_{\mathbf{k}}} \\
& F(K)=-u_{\mathbf{k}} v_{\mathbf{k}}\left(\frac{1}{i \omega_{n}-E_{\mathbf{k}}}-\frac{1}{i \omega_{n}+E_{\mathbf{k}}}\right)
\end{aligned}
$$

where $u_{\mathbf{k}}^{2}=\frac{1}{2}\left(1+\xi_{\mathbf{k}} / E_{\mathbf{k}}\right), v_{\mathbf{k}}^{2}=u_{\mathbf{k}}^{2}-1$. Because bosonic superfluid theories involve a controlled perturbation in the interaction strength, they lead to a clear hierarchy of diagrams and we can restrict attention in the dilute fluid limit to those involving one or at most two Green's functions. The latter constitute the "loops" of the transport approximation.

For transverse response functions, the only diagrams that contribute are those that cannot be divided into two parts by removing one line representing a single-particle propagator. Such diagrams are called proper.
The condensate contributions to a generic correlation function (dependent on a single-particle Green's function) are not proper, and therefore do not contribute to the transverse response functions. It follows that, for a one-loop theory, the transverse component of a generic correlation function is completely determined by diagrams containing only two single-particle Green's functions. For longitudinal correlation functions, other than $L_{11}$, there are no condensate contributions and again the leading order contribution involves two singleparticle Green's functions. In the superfluid phase there are two such Green's functions (the anomalous and normal Green's functions.)

At this bosonic one-loop level we relate these correlation functions to the imaginary time single particle Green's functions in position space, given by $G(x, \tau)$ (normal), $F(x, \tau)$ (anomalous), defined by: $\left\langle T_{\tau} \psi\left(x_{1}\right) \psi^{+}\left(x_{2}\right)\right\rangle=-G\left(x_{1}-x_{2}\right)+n_{0}$ and $\left\langle T_{\tau} \psi\left(x_{1}\right) \psi\left(x_{2}\right)\right\rangle=-F\left(x_{1}-x_{2}\right)+n_{0}$. For convenience, we make the following definitions: the four vector summation $\sum_{K} \equiv-\frac{1}{\beta} \sum_{i \omega_{n}} \int \frac{d^{3} k}{(2 \pi)^{3}}$, the vertex factors $\mathbf{v}_{1}=\left(\frac{\mathbf{k}+\frac{1}{2} \mathbf{q}}{m}\right), \mathbf{v}_{2}=\frac{\mathbf{q}}{2 m}$, and $\mathbf{v}_{3}=$ $\left(\left(i \omega_{n}+i \omega_{m}\right) \frac{\mathbf{k}}{2 m}+i \omega_{n} \frac{\mathbf{k}+\mathbf{q}}{2 m}\right)$. The dissipative parameter $\gamma$ previously introduced also serves to analytically continue the Matsubara frequencies $i \omega_{m}$ to real frequencies $\omega$ via: $i \omega_{m}=\omega+i \gamma$.

With these definitions, the four momentum space correlation functions can be computed. The particle current-particle current correlation function is given by:

$$
\begin{aligned}
& \overleftrightarrow{\chi}_{11}\left(\mathbf{q}, i \omega_{m}\right)=n_{0} \mathbf{v}_{2} \mathbf{v}_{2}[G(Q)+G(-Q)-F(Q)-F(-Q)] \\
& +\sum_{K} \mathbf{v}_{1} \mathbf{v}_{1}[G(K) G(K+Q)-F(K) F(K+Q)] .
\end{aligned}
$$

The particle current-heat current correlation function is:

$$
\begin{aligned}
& \overleftrightarrow{\chi}_{12}\left(\mathbf{q}, i \omega_{m}\right)=\sum_{K} \mathbf{v}_{1} \mathbf{v}_{3} \\
& \times[G(K) G(K+Q)+F(K) F(K+Q)]
\end{aligned}
$$

The heat current-particle current correlation function is:

$$
\begin{aligned}
& \overleftrightarrow{\chi}_{21}\left(\mathbf{q}, i \omega_{m}\right)=\sum_{K} \mathbf{v}_{3} \mathbf{v}_{1} \\
& \times[G(K) G(K+Q)-F(K) F(K+Q)]
\end{aligned}
$$

The heat current-heat current correlation function is:

$$
\begin{aligned}
& \overleftrightarrow{\chi}_{22}\left(\mathbf{q}, i \omega_{m}\right)=\sum_{K} \mathbf{v}_{3} \mathbf{v}_{3} \\
& \times[G(K) G(K+Q)+F(K) F(K+Q)]
\end{aligned}
$$

Our expressions in Eqs. (16-19) contain all possible contributions to the irreducible transverse response functions [18]. Note that, the correlation functions $\overleftrightarrow{\chi}_{12}$ 

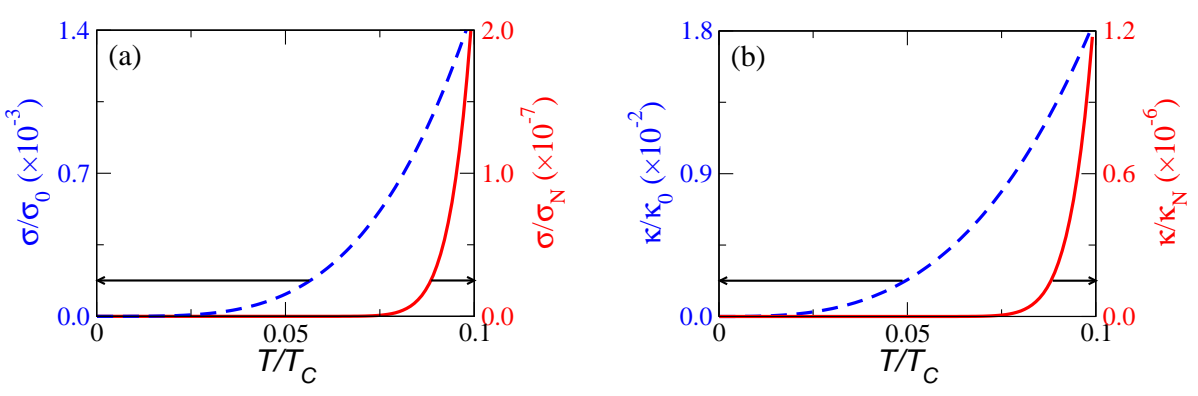

Figure 1: (Color online) The normalized (a) mass and (b) thermal conductivity coefficients for bosonic (dashed blue line) and fermionic (solid red line) superfluids as functions of $T / T_{C}$. The left and right axes are associated with the bosonic and fermionic coefficients, respectively. The fermionic transport coefficients are normalized by the normal state expressions $\sigma_{N}=\sigma(\Delta=$ $0), \kappa_{N}=\kappa(\Delta=0)$. In the bosonic case we define $T / T_{C}=T / \mu^{B}$. In the Bogoliubov approximation $n_{0}$ is the particle number at $T=0$. Thus our calculations are confined to $T / T_{C}<<1$ and so we use the normalization $\sigma_{0}=\frac{\left(2 m \mu^{3}\right)^{1 / 2}}{6 \pi^{2} \gamma}, \kappa_{0}=\frac{\left(2 m \mu^{5}\right)^{1 / 2}}{6 \pi^{2} \gamma}$. Here the mass conductivity corresponds to the $\omega \neq 0$ contribution.

and $\overleftrightarrow{\chi}_{21}$ differ in the relative sign of the contribution from the anomalous Green's functions. It follows that, in order to satisfy the Onsager relation, the anomalous Green's functions must give no contribution to the transport coefficients $L_{12}$ and $L_{21}$. This is confirmed explicitly by direct calculation.

As can be seen, the particle current-particle current correlation function $\left(\overleftrightarrow{\chi}_{11}\right)$ which appears in Eq. (16), unlike all the other $\overleftrightarrow{\chi}_{i j}$, contains a term proportional to the condensate density $n_{0}$. This term is purely longitudinal and of no interest here. In order to ensure charge conservation (via the longitudinal or $f$-sum rule) in the superfluid phase, the condensate requires a consistent treatment, analogous to collective mode effects in fermionic superfluids.

Finally, from the definitions of the transport coefficients, combined with the correlation functions in Eqs. (16-19) and the Bogoliubov Green's functions, the resulting transport coefficients $\eta^{B}$ and $\operatorname{Re} L_{i j}^{B}$ are given by the expressions in Eqs. (4-5).

Figure (1) shows the comparison between the normalized low temperature bosonic and fermionic transport coefficients, corresponding to mass and thermal conductivity. It is clear from the figures that the quasiparticle transport coefficients at these low $T$ differ by several orders of magnitude. This is due to the differences in the quasi-particle excitation spectrum. From an experimental perspective, it appears rather prohibitive to measure very low temperature transport properties of Fermi systems. By contrast it appears Bose systems lend themselves to these low $T$ studies.

\section{Low Temperature Analysis}

In general, the bosonic transport coefficients exhibit power law behavior, whereas the fermionic transport coefficients exhibit an exponentially suppressed response. Explicitly, in the low temperature limits $\left(T<<\mu^{B}, T_{C}\right)$ we find that for bosons

$$
\operatorname{Re} L_{i j}^{B} \rightarrow \frac{2 \pi^{2}}{45 \gamma} m^{1 / 2}\left(\mu^{B}\right)^{i+j-9 / 2} T^{5-j},
$$

whereas for fermions

$$
\begin{aligned}
& \operatorname{Re} L_{11}^{F} \rightarrow \frac{2 g\left(E_{F}\right) p_{F}^{2}}{3 m^{2} \gamma}\left(\frac{2 \pi \Delta_{0}}{T}\right)^{1 / 2} e^{-\Delta_{0} / T}, \\
& \operatorname{Re} L_{22}^{F} \rightarrow \frac{2 g\left(E_{F}\right) p_{F}^{2}}{3 m^{2} \gamma}\left(\frac{2 \pi \Delta_{0}^{3}}{T}\right)^{1 / 2} e^{-\Delta_{0} / T},
\end{aligned}
$$

where $p_{F}$ is the Fermi-momentum, $\Delta_{0}=\Delta(T \rightarrow 0)$, and $g\left(E_{F}\right)$ is the density of states at $E_{F}$. In BCS theory, assuming the chemical potential is of order $\mu^{F} \sim E_{F}$ and with exact particle-hole symmetry, $\operatorname{Re} L_{12}^{F} \rightarrow 0$.

\section{CALCULATION OF $\eta$ AND $\eta / s$}

Kovtun, Son and Starinets (KSS) [1] have made an interesting conjecture concerning the shear viscosity. They conjecture that any relativistic quantum field theory at finite temperature and zero chemical potential has a ratio of shear viscosity to entropy density satisfying the bound $\eta / s \geq \hbar /\left(4 \pi k_{B}\right)$. Despite the construction of certain systems that violate the KSS bound [19], the KSS conjecture has lead to renewed interest in what the perfect fluids in nature are, i.e., those that come as close as possible to minimizing the conjectured bound. It has been shown by KSS that fluids that saturate this bound are those with a dual gravity description.

An interesting feature of the KSS bound is that it is independent of the speed of light $c$. Therefore, a nonrelativistic quantum system is a possible candidate for the perfect fluid. Here we investigate the magnitude of $\eta / s$ arising from quasi-particle transport in the bosonic one-loop and fermionic BCS superfluids.

A variant of the KSS conjecture extends the applicability of the conjectured bound of $\eta / s$ to the 

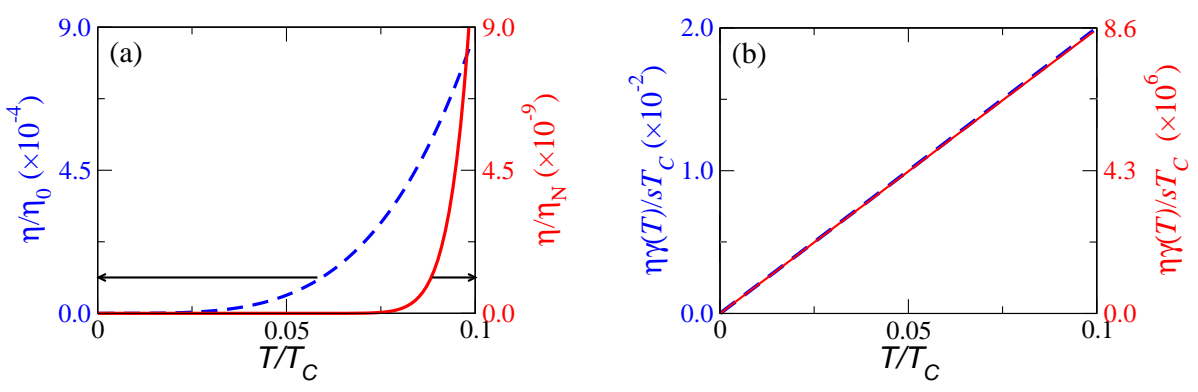

Figure 2: (Color online) (a) The normalized shear viscosity of bosonic (dashed blue line) and fermionic (solid red line) superfluids as functions of $T / T_{C}$. The left and right axes are associated with the bosonic and fermionic coefficients, respectively. The fermionic shear viscosity is normalized by the normal state expression $\eta_{N}=\eta(\Delta=0)$. In the bosonic case we define $T / T_{C}=T / \mu^{B}$. In the Bogoliubov approximation $n_{0}$ is the particle number at $T=0$. Thus our calculations are confined to $T / T_{C}<<1$ and so we use the normalization $\eta_{0}=\frac{\left(2 m^{3} \mu^{5}\right)^{1 / 2}}{15 \pi^{2} \gamma}$. (b) The low temperature limit of the shear viscosity to entropy density ratio. Both limits are linear in $T / \gamma(T)$, and so have the same functional form. The only difference is the associated axes.

case of non-zero chemical potential [20]. If we allow $\mu^{B}, \mu^{F} \neq 0$, then the low temperature entropy limits for bosons and fermions are

$$
\begin{aligned}
& s^{B} \rightarrow \frac{2 \pi^{2}}{45}\left(\frac{m}{\mu^{B}}\right)^{3 / 2} T^{3}, \\
& s^{F} \rightarrow 2 g\left(E_{F}\right)\left(\frac{2 \pi \Delta_{0}^{3}}{T}\right)^{1 / 2} e^{-\Delta_{0} / T} .
\end{aligned}
$$

Similarly the low temperature shear viscosity limits for bosons and fermions are

$$
\begin{aligned}
\eta^{B} & \rightarrow \frac{2 \pi^{2}}{225 \gamma}\left(\frac{m}{\mu^{B}}\right)^{3 / 2} T^{4}, \\
\eta^{F} & \rightarrow \frac{2 g\left(E_{F}\right) p_{F}^{4}}{15 m^{2} \gamma}\left(\frac{2 \pi T}{\Delta_{0}}\right)^{1 / 2} e^{-\Delta_{0} / T} .
\end{aligned}
$$

Depending on the temperature dependence of the quasiparticle lifetimes $\left(\gamma^{-1}\right)$, the bosonic shear viscosity can exhibit an upturn for low temperatures. However, due to the exponentially suppressed term, the fermionic shear viscosity is not expected to exhibit an upturn, regardless of the parameter $\gamma(T)$.

Using the low temperature limits of $s$ and $\eta$ in Eqs. (23-26), we obtain the ratio $\eta / s$ for bosons and fermions:

$$
\begin{aligned}
& \eta^{B} / s^{B} \rightarrow \frac{1}{5} \frac{T}{\gamma}, \\
& \eta^{F} / s^{F} \rightarrow \frac{4}{15}\left(\frac{E_{F}}{\Delta_{0}}\right)^{2} \frac{T}{\gamma},
\end{aligned}
$$

It should be noted that once the entropy density is included, both bosons and fermions exhibit the same $T / \gamma(T)$ dependence in their $\eta / s$ ratios. This derivation confirms the arguments given earlier, namely that systems with a quasi-particle description have a large ratio of $\eta / s$, with the generic form $\eta / s \sim T / \gamma(T)$. An example of the temperature dependence used for $\gamma$ in
${ }^{4} \mathrm{He}$ [11] would predict an upturn in $\eta / s$ at low $T$ for the bosonic superfluid case.

While both bosonic and fermionic cases considered have a similar functional form for the ratio $\eta / s$, the low temperature limits of the entropy and shear viscosity of bosons and fermions are markedly different:

Figure (2) presents a plot of the normalized shear viscosity and the ratio $\eta / s$ for bosonic and fermionic BCS superfluids. While $\eta$ is highly suppressed for the fermionic case (as compared with a bosonic superfluid), in the $\eta / s$ ratio the fermionic contribution is highly enhanced. This is due to the fact that for fermions there are two different energy scales present, $E_{F}$ and $\Delta_{0}$, while for bosons $\mu$ is the only energy scale. Equation (28) shows that there is a factor of the ratio of these two energy scales $\left(E_{F} / \Delta_{0}\right)^{2}$ which appears. This is of course a very large number in the strict BCS limit, which reflects the fact that the entropy in BCS theory is much smaller than the shear viscosity.

\section{BCS-BEC Crossover}

Our primary analysis has been the transport properties of both bosonic and fermionic superfluids in the temperature regime far below the critical temperature. While theories of bosonic superfluidity are restricted to low temperatures, fermionic superfluids can be studied up to $T_{C}$. Equally interesting is the behavior in the normal phase $\left(T>T_{C}\right)$ of superfluids in the presence of stronger attractive interactions, as associated with the crossover from BCS to BEC. In this normal or "pseudogap" phase, pairing persists above $T_{C}$ and is expected to lead to suppressed shear viscosity. With the discovery of the trapped atomic gases one has access to fermionic superfluids with variable attraction, as parameterized by the dimensionless scattering length $1 / k_{F} a$, where $1 / k_{F} a=0$ is the so-called unitary regime. 

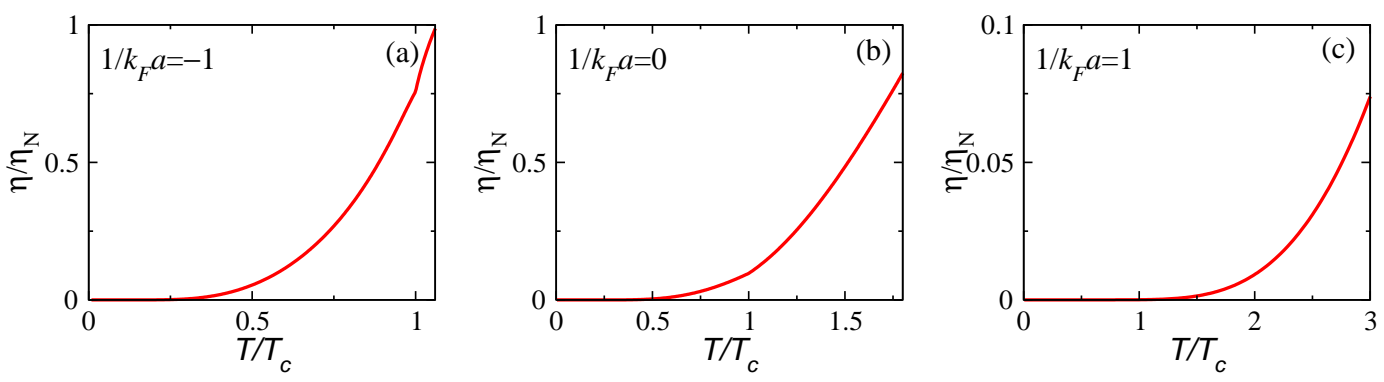

Figure 3: (Color online) The normalized shear viscosity in BCS-BEC crossover theory. The shear viscosity is normalized by the normal state expression $\eta_{N}=\eta\left(T=T^{*}\right)$. The parameters for each plot are (a) $T_{C}=0.12 T_{F}, T^{*}=0.13 T_{F}, 1 / k_{F} a=-1$, (b) $T_{C}=0.26 T_{F}, T^{*}=0.50 T_{F}, 1 / k_{F} a=0$, and (c) $T_{C}=0.21 T_{F}, T^{*}=1.28 T_{F}, 1 / k_{F} a=1$.

One particular BCS-BEC scenario [4] by members of our group (which is explicitly sum-rule consistent) has addressed $\eta^{F}(T)$ theoretically over the entire range of temperatures using as a framework the BCS wave function for the ground state (with arbitrary attraction and self consistent chemical potential). Here one finds that $\eta^{F}=$

$$
\int_{0}^{\infty} d k \frac{k^{6}}{15 \pi^{2} m^{2}}\left(\frac{\xi_{\mathbf{k}}}{E_{\mathbf{k}}}\right)^{2}\left(1-\frac{\Delta_{p g}^{2}}{E_{\mathbf{k}}^{2}}\right)\left(-\frac{\partial f\left(E_{\mathbf{k}}\right)}{\partial E_{\mathbf{k}}}\right) \frac{1}{\gamma},
$$

In this scenario there are two gap functions $\Delta_{s c}$ and $\Delta_{p g}$, where the first represents the order parameter, and the second the contribution to the excitation gap associated with non-condensed pairs. The total gap is given by $\Delta^{2}=$ $\Delta_{s c}^{2}+\Delta_{p g}^{2}$, so that the excitation gap $E_{\mathbf{k}}$ takes the usual BCS form in terms of the full gap $\Delta$. This previous work [4] and the above equation shows that the effect of the non-condensed pairs, associated with the pseudogap $\Delta_{p g}$, is to reduce the shear viscosity. This is due to the fact that when pairs are present there are fewer fermions to contribute to the shear viscosity.

In Fig. (3) we show plots of the normalized shear viscosity for this BCS-BEC crossover scenario. The plots from left to right correspond to passing from the BCS side of unitarity, to unitarity, to the BEC side. The exponential suppression of transport in fermionic superfluids is then reflected in the behavior of $\eta$ : as the gap $\Delta$ increases in size from BCS to BEC the shear viscosity is accordingly suppressed at the lowest $T$. It should be emphasized that the BEC limit still reflects the pairing of fermions and will not coincide with Bogoliubov descriptions of bosons, as the latter involves boson-boson interactions. These are not incorporated into the generalized BCS wave function.

Of particular interest are the observations $[2,3]$ that the unitary superfluids have a shear viscosity which is close to the KSS bound. This makes them very different from the strict BCS superfluids with extremely large $\eta / s$, which we studied earlier in this paper. There are hints from Eq. (28) (which shows that $\eta / s$ for strict
BCS fermions contains a prefactor $\left.\left(T_{F} / T_{C}\right)^{2}\right)$ that as unitarity is approached and $T_{F} / T_{C}$ becomes order one, that $\eta / s$ is significantly reduced relative to the strict BCS case. Indeed, the analogous prefactor $\sim\left(T_{F} / T_{C}\right)^{2}$ is now several orders of magnitude smaller than its counterpart for BCS theory.

As addressed in [4] and seen explicitly in Fig. (3), the presence of the non-condensed pairs via $\Delta_{p g}$ does not affect the exponential suppression of $\eta$ at low temperatures. However, at the same time the entropy acquires an additional bosonic contribution $\left(s=s^{F}+s^{B}\right)$ [21], where $s^{B}$ dominates at low $T$ and is a power of $T$. Thus, the ratio $\eta / s$ will not be a linear function of $T / \gamma$, as was found for strict BCS superfluids; rather, these near-unitary superfluids will exhibit near perfect fluidity.

We summarize this discussion by emphasizing that our theory of BCS-BEC crossover is a theory of fermions and the BEC limit does not include the direct effects of interboson interactions (which give rise to the sound mode excitations of Bogoliubov theory). These sound-mode effects do, of course, appear in the collective modes as the Nambu Goldstone bosons. Such collective modes must be included in some transport coefficients and must not be included in others. More precisely the Nambu Goldstone modes in fermionic superfluids of this type couple to the longitudinal response. They do not couple to the transverse response, of which the shear viscosity is one example. Our previous work on $\eta$ for the BCS-BEC system used a theoretical approach which analytically satisfied the transverse sum rule [4]. A failure to satisfy the sum rules is one of the best internal checks on whether or not, and precisely where, collective modes must be included in a given response function.

\section{CONCLUSIONS}

We have compared the $\omega \rightarrow 0$ mass conductivity, the shear viscosity, and the thermal conductivity in bosonic and fermionic superfluids based on a Kubo formula approach within the one-loop Bogoliubov and closely related BCS approximation. At this level of 
approximation, our work demonstrates the formal (albeit non-quantitative) similarity between the transport behavior of both superfluid types. The transverse response functions do not contain condensate contributions. Similarly for the longitudinal thermoelectric coefficients (aside from the $\omega \equiv 0$ mass conductivity) no condensate contributions appear. Thus, it is appropriate to characterize these coefficients entirely in terms of their quasi-particle contributions, as we have done here.

Of central interest here is the fact that even though the shear viscosity for Bogoliubov and BCS superfluids have dramatically different temperature dependence, their ratios in terms of the entropy density have precisely the same linear $T / \gamma(T)$ dependence (where $\gamma(T)$ is the inverse quasi-particle lifetime) with very different prefactors. When considering the extension of BCS theory to BCS-BEC crossover near unitarity, we find a very different temperature dependence. Here because there are both bosonic and fermionic degrees of freedom, there is no simple $T / \gamma(T)$ scaling. Indeed, due to the suppression of the shear viscosity, it appears that unitary Fermi gases are a candidate for nearly perfect fluids.

We stress that pure bosonic or Bogoliubov theories of superfluidity have a structure not exhibited in the BCS-BEC crossover; this difference arises due to the soft dispersion relation present in the long wavelength limit of Bogoliubov theory. Similarly, inter-boson interactions are not directly present at the level of a BCS-based theory of unitarity. Here the dominant many body physics is an attraction between fermions, as distinct from boson-boson interactions. Recent experiments [22] seem to confirm this exponential suppression in the low temperature shear viscosity as unitarity is approached.

We end by noting that essentially all reasonable models for the temperature dependence of the transport lifetime will give an upturn in $\eta / s$ at low $T$, but not, for the case of fermions, in $\eta$ itself. This appears consistent with the observed differences between helium-3 and helium-4 superfluids [23].

This work is supported by NSF-MRSEC Grant 0820054. We thank Adam Rançon for many helpful conversations.

[1] P. K. Kovtun, D. T. Son, and A. O. Starinets, Phys. Rev. Lett. 94, 111601 (2005).

[2] C. Cao, E. Elliott, J. Joseph, H. Wu, J. Petricka, T. Schäfer, and J. E. Thomas, Science 331, 58 (2010).

[3] C. Cao, E. Elliott, H. Wu, and J. E. Thomas, New J. Phys. 13, 075007 (2011).

[4] H. Guo, D. Wulin, C.-C. Chien, and K. Levin, Phys. Rev. Lett. 107, 020403 (2011).

[5] M. Müller, J. Schmalian, and L. Fritz, Phys. Rev. Lett. 103, 025301 (2009).
[6] J. D. Rameau, T. J. Reber, H.-B. Yang, S. Akhanjee, G. D. Gu, P. D. Johnson, and S. Campbell, Phys. Rev. B 90, 134509 (2014).

[7] V. K. Wong and H. Gould, Ann. Phys. 83, 252 (1974).

[8] E. Talbot and A. Griffin, Ann. Phys. 151, 71 (1983).

[9] L. P. Roobol, P. Remeijer, S. C. Steel, R. Jochemsen, V. S. Shumeiko, and G. Frossati, Phys. Rev. Lett. 79, 685 (1997).

[10] A. D. B. Woods and A. C. Hollis Hallett, Can. J. Phys. 41, 596 (1963).

[11] I. M. Khalatnikov, An Introduction to the Theory of Superfluidity (Perseus, New York, 2000).

[12] A. Griffin, T. Nikuni, and E. Zaremba, Bose-Condensed Gases at Finite Temperatures (Cambridge University Press, Cambridge, 2009).

[13] B. Bradlyn, M. Goldstein, and N. Read, Phys. Rev. B 86, 245309 (2012).

[14] L. P. Kadanoff and P. C. Martin, Ann. Phys. 24, 419 (1963).

[15] L. P. Kadanoff and P. C. Martin, Phys. Rev. 124, 670 (1961).

[16] M. A. Shahzamanian and H. Yavary, Ann. Phys. 321, 1063 (2006).

[17] P. K. Kovtun, D. T. Son, A. O. Starinets, J. High Energy Phys. 10, 064 (2003).

[18] A. Griffin, Excitations in a Bose Condensed Liquid (Cambridge University Press, Cambridge, 1993).

[19] A. Cherman, T. D. Cohen, and P. M. Hohler, J. High Energy Phys. 02, 026 (2008).

[20] D. T. Son and A. O. Starinets, Ann. Rev. Nucl. Part. Sci. 57, 95 (2007).

[21] Q. Chen, J. Stajic, S. Tan, and K. Levin, Phys. Reports 412, 1 (2005).

[22] J. A. Joseph, E. Elliott, and J. E. Thomas arXiv:1410.4835 [cond-mat.quant-gas].

[23] H. Guo, D. Wulin, C.-C. Chien, and K. Levin, New J. Phys. 13, 075011 (2011). 